\title{
RESEARCH
}

\section{On the cohomology of the space of seven points in general linear position}

\author{
Olof Bergvall* (1)
}

${ }^{*}$ Correspondence:
olof.bergvall@hig.se
Department of Electronics,
Mathematics, and Natural
Sciences, University of Gävle,
Gävle, Sweden

"Correspondence: olof.bergvall@hig.se Mathematics, and Natural Gävle, Sweden

\begin{abstract}
We determine the cohomology groups of the space of seven points in general linear position in the projective plane as representations of the symmetric group on seven elements by making equivariant point counts over finite fields. We also comment on the case of eight points.
\end{abstract}

Keywords: Configurations of points, Moduli spaces, Ėtale cohomology, de Rham cohomology, Group actions, Representations of finite groups

Mathematics Subject Classification: $14 \mathrm{~N} 20$ (primary)14H10, 14J10, 14J45, 14F20, 14F40, 14L30 (secondary)

\section{Introduction}

Given a variety $X$, it is very natural to consider $m$-tuples $\left(P_{1}, \ldots, P_{m}\right)$ of points on $X$. If no condition is placed on the tuples, the space of these tuples is $X^{m}$ and the space of tuples such that the points are distinct is the configuration space $\operatorname{Conf}_{m}(X)$. If the order of the points is irrelevant, one instead arrives at the symmetric product $S^{m}(X)$ and the unordered configuration space $\operatorname{UConf}_{m}(X)$, respectively. The ordered and unordered spaces are naturally related through the action of the symmetric group $S_{m}$ permuting the points.

It is both possible and interesting to pose more refined requirements on the $m$-tuples; For instance, tuples of points in general position, i.e., such that there is no "unexpected" subvariety containing the points, have been studied extensively in the classical literature. See the book [9] of Dolgachev and Ortland for a modern account on this topic and further references. Tuples of points in general position also have close connections to various moduli spaces of curves, surfaces, and abelian varieties; see, e.g., [1,3,19]. We also mention that spaces of $m$-tuples in general position in $\mathbb{P}^{2}$ have been studied from a cohomological point of view by Gounelas and the author when $m$ is at most seven; see [3-5]. The techniques employ both point counts over finite fields and purity arguments, two topics that are discussed further in the present paper.

In $\mathbb{P}^{n}$, it is also natural to consider $m$-tuples of points in general linear position. In other words, we require that no subset of $n+1$ points should lie on a hyperplane. We denote the space of $m$-tuples of points in $\mathbb{P}^{n}$ which are in general linear position by $\operatorname{Conf}_{m}^{\text {glp }}\left(\mathbb{P}^{n}\right)$ and

(c) The Author(s) 2020. This article is licensed under a Creative Commons Attribution 4.0 International License, which permits use, sharing, adaptation, distribution and reproduction in any medium or format, as long as you give appropriate credit to the original author(s) and the source, provide a link to the Creative Commons licence, and indicate if changes were made. The images or other third party material in this article are included in the article's Creative Commons licence, unless indicated otherwise in a credit line to the material. If material is not included in the article's Creative Commons licence and your intended use is not permitted by statutory regulation or exceeds the permitted use, you will need to obtain permission directly from the copyright holder. To view a copy of this licence, visit http://creativecommons.org/licenses/by/4.0/. 
its quotient by PGL $(n+1)$ by $\mathcal{C}_{n, m}^{\text {glp }} \cdot{ }^{1}$ In fact, $\operatorname{Conf}_{m}^{\text {glp }}\left(\mathbb{P}^{n}\right) \cong \operatorname{PGL}(n+1) \times \mathcal{C}_{n, m}^{\text {glp }}$, so for many purposes it is merely a matter of convenience (or preference) which one to use. In this work, the spaces $\mathcal{C}_{n, m}^{\mathrm{glp}}$ are more convenient since they (sometimes) satisfy certain cohomological purity properties which the spaces $\operatorname{Conf}_{m}^{\text {glp }}\left(\mathbb{P}^{n}\right)$ do not satisfy. Also, the spaces $\mathcal{C}_{n, m}^{\text {glp }}$ have received considerable attention classically; see, e.g., the work of Dolgachev and Ortland [9]. From a more modern perspective, the spaces $\mathcal{C}_{n, m}^{\mathrm{glp}}$ can be viewed as natural generalizations of the moduli spaces $\mathcal{M}_{0, n}$ of $n$-pointed rational curves. In fact, sending a $(n+3)$-tuple of points in $\mathbb{P}^{n}$ to the isomorphism class of the rational normal curve passing through them gives an isomorphism $\mathcal{C}_{n, n+3}^{\text {glp }} \cong \mathcal{M}_{0, n+3}$. The spaces $\mathcal{C}_{n, m}^{\text {glp }}$, especially in the case $n=2$, have also been studied extensively from the perspective of coding theory starting with the work of Glynn [11] and continued in the work of Iampolskaia, Skorobogatov, and Sorokin [13] and Kaplan et al. [14]. In particular, the number $\left|\mathcal{C}_{2, m}^{\text {glp }}\left(\mathbb{F}_{q}\right)\right|$ of $m$-tuples of points in general linear position in the projective plane over the finite field $\mathbb{F}_{q}$ with $q$ elements is known for all $q$ and $m<10$ through these works.

Through Lefschetz's trace formula, these point counts determine the Euler characteristics of the spaces $\mathcal{C}_{n, m}^{\text {glp }}$. By also considering the action of the group $S_{m}$, these Euler characteristics can be decomposed into virtual representations of $S_{m}$. From an arithmetic point of view, this allows one to obtain more refined enumerative information about the counted objects. For an example in this direction, see the recent paper of Das [6]. From a more topological or representation-theoretic perspective, the $S_{m}$-equivariant information encodes cohomological information about quotients of $\mathcal{C}_{n, m}^{\mathrm{glp}}$ by subgroups of $S_{m}$; in particular, one obtains the Euler characteristic of the "unordered space" $\mathcal{C}_{n, m}^{\text {glp }} / S_{m}$.

If $m \leq 6$, it has been shown through the work of Gounelas and the author [5] and Das and O'Connor [7] that $\mathcal{C}_{2, m}^{\text {glp }}$ satisfies a strong condition called minimal purity. This purity condition implies that the $S_{m}$-equivariant point counts of $\mathcal{C}_{2, m}^{\text {glp }}$ do not only determine the Euler characteristic as a virtual representation of $S_{m}$ but actually determine each cohomology group $H^{i}\left(\mathcal{C}_{2, m}^{\text {glp }}\right)$ as a representation of $S_{m}$.

The purpose of the present paper is to extend these results to the case of seven points. Our main result is the following:

Theorem 1.1 ${ }^{2}$ The cohomology groups $H^{k}\left(\mathcal{C}_{2,7}^{\text {glp }}\right)$ of the space $\mathcal{C}_{2,7}^{\text {glp }}$ of seven points in general linear position are pure of Hodge-Tate type $(k, k)$ (i.e., $H^{k}\left(\mathcal{C}_{2,7}^{\mathrm{glp}}\right)$ consists entirely of $(k, k)$ classes). The cohomology groups are described explicitly in Table 2 as representations of the symmetric group $S_{7}$.

We prove Theorem 1.1 essentially by first relating the space $\mathcal{C}_{2,7}^{\text {glp }}$ to the complement of a certain toric arrangement (the cohomology of such complements has been studied extensively) and then counting points of $\mathcal{C}_{2,7}^{\text {glp }}$ over finite fields and applying Lefschetz's trace formula.

We also discuss the case of eight points, in which case, we are able to prove the following:

Theorem 1.2 The cohomology groups $H^{k}\left(\mathcal{C}_{2,8}^{\mathrm{gp}}\right)$ of the space $\mathcal{C}_{2,8}^{\mathrm{gp}}$ of eight points in general position are pure of Hodge-Tate type $(k, k)$.

\footnotetext{
${ }^{1}$ To avoid technical subtleties, we assume $m \geq n+2$.

${ }^{2}$ In most of the paper, we work over a field of positive (odd) characteristic and use compactly supported étale cohomology. One can also interpret the result in characteristic 0 and using ordinary (de Rham) cohomology via a standard argument using Artin's comparison theorem (as well as constructibility and base change) and Poincaré duality.
} 
Our method fails to prove the analogous result for the space of eight points in general linear position. This suggests that the transition in the mixed Hodge structure from minimal purity to more general behavior occurs between seven and eight points for points in general linear position, while the transition for points in general position seems to be between eight and nine. To decide whether this is actually the case would of course be very interesting. More generally, we have the following question:

Question 1.3 For which integers $m$ and $n$ and for which generality conditions $g c$ is the space $\mathcal{C}_{n, m}^{g c}$ of m-tuples of points in $\mathbb{P}^{n}$ satisfying these conditions minimally pure?

We also remark that Propositions 3.1 and 4.1, especially the proofs, are closely related to moduli of surfaces with anticanonical cycles. Such moduli spaces have been studied quite extensively, e.g., in the work [18] and, more recently, by Gross, Hacking, and Keel [12]. The present work sheds new light on the cohomology of some of these spaces. We also expect the present paper to be of use in the pursuit of counting pairs of curves with prescribed intersection; see, e.g., the recent paper of Kaplan and Matei [15].

\section{Background}

Let $p$ be an $\operatorname{odd}^{3}$ prime number, let $n \geq 1$ be an integer, and let $q=p^{n}$. Let $\mathbb{F}_{q}$ be a finite field with $q$ elements, let $\mathbb{F}_{q^{m}}$ be a degree- $m$ extension of $\mathbb{F}_{q}$, and let $\overline{\mathbb{F}}_{q}$ be an algebraic closure of $\mathbb{F}_{q}$. Let $X$ be a scheme defined over $\mathbb{F}_{q}$, and let $F$ denote its geometric Frobenius endomorphism. Finally, let $\ell$ be a prime number different from $p$, and let $H_{\mathrm{et}, c}^{k}\left(X, \mathbb{Q}_{\ell}\right)$ denote the $k$-th compactly supported étale cohomology group of $X$ with coefficients in $\mathbb{Q}_{\ell}$.

If $\Gamma$ is a finite group acting on $X$ by $\mathbb{F}_{q}$-rational automorphisms, then each cohomology group $H_{\mathrm{et}, c}^{k}\left(X, \mathbb{Q}_{\ell}\right)$ is a $\Gamma$-representation. Lefschetz's trace formula allows us to obtain information about these representations by counting the number of fixed points of $F \sigma$ for different $\sigma \in \Gamma$.

Theorem 2.1 (Lefschetz's trace formula) Let $X$ be a separated scheme of finite type over $\mathbb{F}_{q}$ with Frobenius endomorphism $F$, and let $\sigma$ be a rational automorphism of $X$ of finite order. Then

$$
\left|X\left(\overline{\mathbb{F}}_{q}\right)^{F \sigma}\right|=\sum_{k \geq 0}(-1)^{k} \cdot \operatorname{Tr}\left(F \sigma, H_{\mathrm{e}, t, c}^{k}\left(X\left(\overline{\mathbb{F}}_{q}\right), \mathbb{Q}_{\ell}\right)\right)
$$

where $\bar{X}^{F \sigma}$ denotes the fixed point set of Fo.

Under the assumptions of the theorem, the number $\left|X\left(\overline{\mathbb{F}}_{q}\right)^{F \sigma}\right|$ only depends on the conjugacy class of $\sigma$.

Let $R(\Gamma)$ denote the representation ring of $\Gamma$, and let the compactly supported $\Gamma$ equivariant Euler characteristic of $X$ be defined as the virtual representation

$$
\operatorname{Eul}_{X, c}^{\Gamma_{1}}=\sum_{k \geq 0}(-1)^{k} \cdot H_{\mathrm{et}, c}^{k}\left(X, \mathbb{Q}_{\ell}\right) \in R(\Gamma) .
$$

\footnotetext{
${ }_{3}^{3}$ In this section, there would be no harm in allowing $p=2$. However, our applications require $p$ to be odd; see [14] for
} some details on what goes wrong for $p=2$. See also [20] for some details on what can be salvaged even when $p=2$. 
By evaluating $\operatorname{Eul}_{X, c}^{\Gamma}$ at an element $\sigma \in \Gamma$ we mean

$$
\operatorname{Eul}_{X, c}^{\Gamma}(\sigma)=\sum_{k \geq 0}(-1)^{k} \cdot \operatorname{Tr}\left(\sigma, H_{\text {ét }, c}^{k}\left(X, \mathbb{Q}_{\ell}\right)\right) \in \mathbb{Z}
$$

Note in particular that $\operatorname{Eul}_{X, c}^{\Gamma}$ (id) is the ordinary Euler characteristic of $X$. By character theory, $\operatorname{Eul}_{X, c}^{\Gamma_{n}}$ is completely determined by computing $\operatorname{Eul}_{X, c}^{\Gamma}(\sigma)$ for a representative $\sigma$ of each conjugacy class of $\Gamma$.

Thus, phrased in these terms, Lefschetz's trace formula says that the $\Gamma$-equivariant Euler characteristic of $X$ is determined by counting fixed points of $F \sigma$ for a representative $\sigma$ of each conjugacy class of $\Gamma$. If we make stronger assumptions on $X$ we can say more about its cohomology from these equivariant point counts.

Definition 2.2 (Dimca and Lehrer [8]) Let $X$ be an irreducible and separated scheme of finite type over $\overline{\mathbb{F}}_{q}$. The scheme $X$ is called minimally pure if $F$ acts on $H_{\text {ét,c }}^{k}\left(X, \mathbb{Q}_{\ell}\right)$ by multiplication by $q^{k-\operatorname{dim}(X)}$.

Examples of minimally pure varieties include complements of hyperplane arrangements, complements of toric arrangements, and quotients of reductive groups by maximal tori. For further examples, see $[8,16,17]$.

A pure dimensional and separated scheme $X$ of finite type over $\overline{\mathbb{F}}_{q}$ is minimally pure if, for any collection $\left\{X_{1}, \ldots, X_{r}\right\}$ of irreducible components of $X$, the irreducible scheme $X_{1} \backslash\left(X_{2} \cup \cdots \cup X_{r}\right)$ is minimally pure.

We define the compactly supported $\Gamma$-equivariant Poincaré polynomial of $X$ as

$$
P_{X, c}^{\Gamma}(t)=\sum_{k \geq 0} H_{\mathrm{et}, c}^{k}\left(X, \mathbb{Q}_{\ell}\right) \cdot t^{k} \in R(\Gamma)[t],
$$

and we introduce the notation

$$
P_{X, c}^{\Gamma}(\sigma)(t)=\sum_{k \geq 0} \operatorname{Tr}\left(\sigma, H_{\text {ét, } c}^{k}\left(X, \mathbb{Q}_{\ell}\right)\right) \cdot t^{k} \in \mathbb{Z}[t] .
$$

Thus, if $X$ is minimally pure, then the eigenvalues of $F \sigma$ on $H_{\text {ét, } c}^{k}\left(X, \mathbb{Q}_{\ell}\right)$ are of the form $\zeta q^{k-\operatorname{dim}(X)}$ for some root of unity $\zeta$. Thus, a term $q^{k-\operatorname{dim}(X)}$ in $\left|X^{F \circ \sigma}\right|$ can only come from $H_{\text {ét, }}^{k}\left(X, \mathbb{Q}_{\ell}\right)$, and we can determine the $\Gamma$-equivariant Poincaré polynomial of $X$ via the relation

$$
\operatorname{Eul}_{X, c}^{\Gamma}(\sigma)=q^{-2 \operatorname{dim}(X)} \cdot P_{X, c}^{\Gamma}(\sigma)\left(-q^{2}\right)
$$

In particular we see that, if $X$ is minimally pure, then we can determine the cohomology groups of $X$ by counting points over finite fields.

Using Lefschetz's trace formula, one may interpret Eq. 2.1 as an equality of integers. On the other hand, we may also view the above expression as a polynomial in $q$. We then see that the point count is polynomial and that the coefficients of this polynomial are given by the values of the characters of the $\Gamma$-representations $H_{\mathrm{e} t, c}^{k}\left(X, \mathbb{Q}_{\ell}\right)$. 


\section{The space of seven points}

Let $T=\left(P_{1}, \ldots, P_{7}\right)$ be a septuple of points in $\mathbb{P}^{2}$. We say that $T$ is in general linear position if no three of the points lie on a line. We denote the space of septuples in $\mathbb{P}^{2}$ in general linear position, up to projective equivalence, by $\mathcal{C}_{2,7}^{\text {glp }}$.

Proposition 3.1 The space $\mathcal{C}_{2,7}^{\text {glp }}$ of seven points in the plane in general linear position is minimally pure.

Proof The idea of the proof is as follows: We shall construct a $\mathbb{F}_{q}$-rational and $S_{7}$ equivariant finite cover $\varphi: X \rightarrow \mathcal{C}_{2,7}^{\text {glp }}$ such that $X$ is minimally pure. A finite cover $\varphi: X \rightarrow \mathcal{C}_{2,7}^{\text {glp }}$ induces an $\mathbb{F}_{q}$-rational inclusion $H_{\text {ét,c }}^{k}\left(\mathcal{C}_{2,7}^{\text {glp }}, \mathbb{Q}_{\ell}\right) \hookrightarrow H_{\text {ét, }}^{k}\left(X, \mathbb{Q}_{\ell}\right)$. Thus, the image of $H_{\text {et }, c}^{k}\left(\mathcal{C}_{2,7}^{\text {glp }}, \mathbb{Q}_{\ell}\right)$ is preserved by the Frobenius. Since $X$ is minimally pure, $F$ acts on $H_{\text {et }, c}^{k}\left(X, \mathbb{Q}_{\ell}\right)$ with all eigenvalues equal to $q^{k-\operatorname{dim}(X)}$. We then see that $F$ also acts with all eigenvalues equal to $q^{k-\operatorname{dim}(X)}$ on the subspace $H_{\text {ét, }}^{k}\left(\mathcal{C}_{2,7}^{\text {glp }}, \mathbb{Q}_{\ell}\right)$, i.e., that $\mathcal{C}_{2,7}^{\text {glp }}$ is minimally pure.

To construct the space $X$ we observe that, if we blow up $\mathbb{P}^{2}$ in seven points $P_{1}, \ldots, P_{7}$ in general linear position, we obtain a weak Del Pezzo surface $S$ of degree 2 marked with seven (-1)-curves $E_{1}, \ldots, E_{7}$. Together with $L$, the strict transform of a line, these curves constitute a basis $B$ for the Picard group of $S$. Such a basis for $\operatorname{Pic}(S)$ (i.e., one arising in this way from a blow-up) is called a geometric marking. Let $\pi: S \rightarrow \mathbb{P}^{2}$ denote the blow-down morphism. We rigidify the situation further by marking $S$ with an anticanonical curve $C$, i.e., a curve whose class is $3 L-E_{1}-\cdots-E_{7}$. The curve $A=\pi(C)$ has degree 3 . Thus, if $A$ is reducible, it will consist of two components $A_{1}$ and $A_{2}$ of degree 1 and 2, respectively.

Let $X$ be the space of triples $(S, B, C)$ such that $S$ is a weak Del Pezzo surface of degree 2, $B$ is a geometric marking of $S$, and $C$ is an anticanonical curve on $S$ such that $A=\pi(C)$ is reducible and the degree 1 component $A_{1}$ of $A$ contains two of the points $P_{1}, \ldots, P_{7}$. Two triples $(S, B, C)$ and $\left(S^{\prime}, B^{\prime}, C^{\prime}\right)$ are equivalent if there is an isomorphism $S \rightarrow S^{\prime}$ taking the ordered basis $B$ to $B^{\prime}$ and the curve $C$ to $C^{\prime}$. The morphism $\varphi: X \rightarrow \mathcal{C}_{2,7}^{\text {glp }}$ sending a triple $(S, B, C)$ to the septuple $\left(P_{1}, \ldots, P_{7}\right)$ defined by the blow-down of $B$ is clearly $\mathbb{F}_{q}$-rational, finite (of degree 21), and $S_{7}$-equivariant. It thus remains to show that $X$ is minimally pure.

Note that either the curve $A_{1}$ will intersect the degree 2 component $A_{2}$ transversally at two points (see Fig. 1a-c) or $A_{1}$ will be tangent to $A_{2}$ at some point (see Fig. $1 \mathrm{~d}$, e). The points of intersection may or may not coincide with some of the points $P_{1}, \ldots, P_{7}$ (see Fig. 1). Let $X^{\text {gen }}$ denote the subspace of $X$ consisting of triples $(S, B, C)$ such that $A_{1}$ and $A_{2}$ intersect transversally and let $X^{\tan }$ be the subspace of $X$ consisting of triples such that $A_{1}$ is a tangent to $A_{2}$. We then have that $X$ is the disjoint union

$$
X=X^{\mathrm{gen}} \sqcup X^{\tan } .
$$

The next step is to show that $X^{\text {gen }}$ is isomorphic to a disjoint union of complements of toric arrangements in algebraic tori $T_{i}$ and that $X^{\tan }$ is isomorphic to a disjoint union of projectivizations of complements of hyperplane arrangements in vector spaces $V_{i}$ in such a way that $V_{i}$ can naturally be identified with the tangent space of $T_{i}$ at the identity. This follows exactly as in Sect. 1 of [19] (see in particular Propositions 1.13, 1.15, and 1.17), with the very small modification that we need to remove the conditions corresponding to disallowing more than five of the points $P_{1}, \ldots, P_{7}$ from lying on a conic. See also [5] (Theorem 5.1) for a slightly different account of the same ideas. 


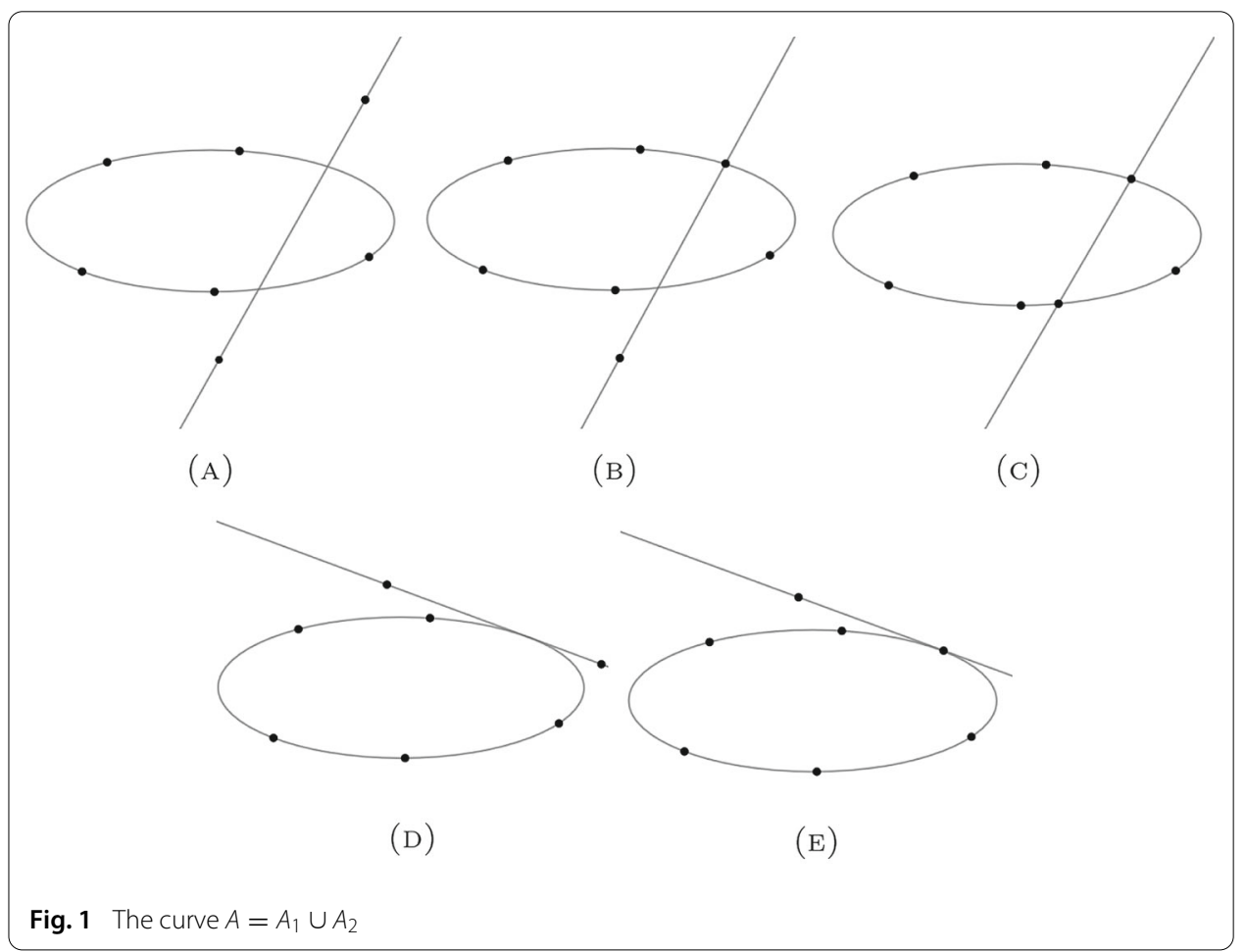

It now follows directly from Lemma 3.6 of [19] that there is a short exact sequence

$$
0 \rightarrow H_{\mathrm{et}, c}^{k}\left(X, \mathbb{Q}_{\ell}\right) \rightarrow H_{\mathrm{e} t, c}^{k}\left(X^{\mathrm{gen}}, \mathbb{Q}_{\ell}\right) \rightarrow H_{\mathrm{e} t, c}^{k-1}\left(X^{\mathrm{tan}}, \mathbb{Q}_{\ell}\right)(-1) \rightarrow 0 .
$$

In particular, it follows that there is an inclusion

$$
H_{\mathrm{et}, c}^{k}\left(X, \mathbb{Q}_{\ell}\right) \hookrightarrow H_{\mathrm{et}, c}^{k}\left(X^{\mathrm{gen}}, \mathbb{Q}_{\ell}\right) .
$$

Complements of toric arrangements are well known to be minimally pure (see, e.g., [8]), so it follows that also $X$ is minimally pure. This completes the proof.

By Proposition 3.1, we can determine the cohomology groups of $\mathcal{C}_{2,7}^{\text {glp }}$ as representations of $S_{7}$ by counting fixed points of $F \sigma$ for each $\sigma \in S_{7}$ and then applying Lefschetz's trace formula. Since the number $\left|\left(\mathcal{C}_{2,7}^{\text {glp }}\right)^{F \sigma}\right|$ only depends on the conjugacy class of $\sigma$, we only have to do one computation for each of the 15 conjugacy classes of $S_{7}$. We denote the conjugacy class of $\sigma$ by $[\sigma]$. Recall also that the conjugacy class $[\sigma]$ is determined by the cycle type of $\sigma$. We denote cycle types by $7^{n_{7}} 6^{n_{6}} \cdots 2^{n_{2}} 1^{n_{1}}$ (with the convention of omitting $m^{n_{m}}$ if $n_{m}=0$ and writing $m$ instead of $m^{n_{m}}$ if $n_{m}=1$ ).

The necessary computations can be deduced quite straightforwardly from the corresponding computations in [4]. We therefore omit the details in most cases and simply record the results in Table 1. However, to illustrate the nature of the computations, at least in one of the simpler cases, we include the following lemma:

Lemma 3.2 If $[\sigma]=[7]$, then $\left|\left(\mathcal{C}_{2,7}^{\text {glp }}\right) F \sigma\right|=q^{6}+q^{4}+q^{3}+q^{2}+1$.

Proof Since the result is independent of the particular representative of the conjugacy class, we pick $\sigma$ so that $\sigma^{-1}=(1234567)$. Let $X=\left(\mathbb{P}^{2}\right)^{7}$, let $\Delta$ denote the subspace of $X$ consisting of septuples $\left(P_{1}, \ldots, P_{7}\right)$ such that at least three of the points lie on a line, and let 
Table $1 S_{7}$-equivariant point counts of the space of seven points in general linear position in $\mathbb{P}^{2}$

\begin{tabular}{ll}
\hline$[\sigma]$ & $\left|\left(\mathcal{C}_{2,7}^{g \mid p}\right)^{F \sigma}\right|$ \\
\hline 7 & $q^{6}+q^{4}+q^{3}+q^{2}+1$ \\
61 & $q^{6}+q^{5}+q^{4}-q^{2}$ \\
52 & $q^{6}+q^{4}-q^{3}-q$ \\
$51^{2}$ & $q^{6}+2 q^{5}+3 q^{4}+3 q^{3}+2 q^{2}+q$ \\
43 & $q^{6}-q^{5}-q^{4}+q^{3}$ \\
421 & $q^{6}-3 q^{4}+2$ \\
$41^{3}$ & $q^{6}+2 q^{5}-q^{4}-2 q^{3}$ \\
$3^{2} 1$ & $q^{6}-q^{5}-q^{4}-8 q^{3}+9 q^{2}+6 q+18$ \\
$32^{2}$ & $q^{6}-q^{5}-q^{4}+q^{3}$ \\
$321^{2}$ & $q^{6}-q^{5}-q^{4}+q^{3}$ \\
$31^{4}$ & $q^{6}-q^{5}-q^{4}+q^{3}$ \\
$2^{3} 1$ & $q^{6}-2 q^{5}-11 q^{4}+18 q^{3}+38 q^{2}-36 q-48$ \\
$2^{2} 1^{3}$ & $q^{6}-4 q^{5}-q^{4}+16 q^{3}-6 q^{2}-12 q+6$ \\
$21^{5}$ & $q^{6}-10 q^{5}+41 q^{4}-86 q^{3}+90 q^{2}-36 q$ \\
$1^{7}$ & $q^{6}-28 q^{5}+323 q^{4}-1952 q^{3}+6462 q^{2}-11004 q+7470$ \\
\hline
\end{tabular}

$U=X \backslash \Delta$. The decomposition $X=U \sqcup \Delta$ is stable both under the action of the Frobenius and under the action of $S_{7}$ and $\left(\mathcal{C}_{2,7}^{\text {glp }}\right)^{F \sigma}=U^{F \sigma} / \operatorname{PGL}\left(3, \mathbb{F}_{q}\right)$. Since $\left|U^{F \sigma}\right|=\left|X^{F \sigma}\right|-\left|\Delta^{F \sigma}\right|$, we can obtain the result by counting $X^{F \sigma}$ and $\Delta^{F \sigma}$.

A septuple $\left(P_{1}, \ldots, P_{7}\right)$ is fixed by $F \sigma$ if and only if $F$ acts as $\sigma^{-1}=(1234567)$, i.e.,

$$
F P_{1}=P_{2}, \quad F P_{2}=P_{3}, \quad \ldots \quad F P_{6}=P_{7}, \quad F P_{7}=P_{1} .
$$

We may thus obtain such a septuple by choosing a point $P_{1} \in \mathbb{P}^{2}$ which is defined over $\mathbb{F}_{q^{7}}$ but not over $\mathbb{F}_{q}$ and then acting by $F$. Thus, to count $\left|X^{F \sigma}\right|$, we need to count $\left|\mathbb{P}^{2}\left(\mathbb{F}_{q^{7}}\right)\right|$ and $\left|\mathbb{P}^{2}\left(\mathbb{F}_{q}\right)\right|$ and take the difference:

$$
\left|X^{F \sigma}\right|=\left|\mathbb{P}^{2}\left(\mathbb{F}_{q^{7}}\right)\right|-\left|\mathbb{P}^{2}\left(\mathbb{F}_{q}\right)\right|=\left(q^{14}+q^{7}+1\right)-\left(q^{2}+q+1\right)=q^{14}+q^{7}-q^{2}-q .
$$

To determine $\left|\Delta^{F \sigma}\right|$ we observe that, if $\left(P_{1}, \ldots, P_{7}\right)$ is a septuple in $X^{F \sigma}$ such that three of the points lie on a line $L$, then $L$ must be defined over $\mathbb{F}_{q}$ and $L$ must contain all seven points $P_{1}, \ldots, P_{7}$ (see Lemma 4 of [4] for some elaboration on this fact). Thus, to determine $\left|\Delta^{F \sigma}\right|$ we should count the number of lines $L \subset \mathbb{P}^{2}$ defined over $\mathbb{F}_{q}$ and the number of ways to pick a point $P_{1}$ on $L$ such that $P_{1}$ is defined over $\mathbb{F}_{q^{7}}$ but not over $\mathbb{F}_{q}$. The number of $\mathbb{F}_{q}$-lines $L$ in $\mathbb{P}^{2}$ is $q^{2}+q+1$ and the number of ways to pick $P_{1}$ on $L$ is $\left(q^{7}+1\right)-(q+1)=q^{7}-q$. We thus obtain the result

$$
\left|\Delta^{F \sigma}\right|=\left(q^{2}+q+1\right)\left(q^{7}-q\right)=q^{9}+q^{8}+q^{7}-q^{3}-q^{2}-q .
$$

We get

$$
\left|X^{F \sigma}\right|-\left|\Delta^{F \sigma}\right|=q^{14}-q^{9}-q^{8}+q^{3}
$$

and finally

$$
\left|\left(\mathcal{C}_{2,7}^{\mathrm{glp}}\right) F \sigma\right|=\frac{\left|X^{F \sigma}\right|-\left|\Delta^{F \sigma}\right|}{\left|\operatorname{PGL}\left(3, \mathbb{F}_{q}\right)\right|}=\frac{q^{14}-q^{9}-q^{8}+q^{3}}{\left(q^{2}+q+1\right)\left(q^{3}-q\right)\left(q^{3}-q^{2}\right)}=q^{6}+q^{4}+q^{3}+q^{2}+1 .
$$


Table 2 The cohomology groups of $\mathcal{C}_{2,7}^{g / p}$ as representations of $S_{7}$

\begin{tabular}{llllllllllllllll}
\hline & 7 & 6 & 52 & $51^{2}$ & 43 & 421 & $41^{3}$ & $3^{2} 1$ & $32^{2}$ & $321^{2}$ & $31^{4}$ & $2^{3} 1$ & $2^{2} 1^{3}$ & $21^{5}$ & $1^{7}$ \\
\hline$H^{0}$ & 1 & 0 & 0 & 0 & 0 & 0 & 0 & 0 & 0 & 0 & 0 & 0 & 0 & 0 & 0 \\
$H^{1}$ & 0 & 0 & 1 & 0 & 1 & 0 & 0 & 0 & 0 & 0 & 0 & 0 & 0 & 0 & 0 \\
$H^{2}$ & 0 & 1 & 1 & 3 & 1 & 3 & 1 & 3 & 1 & 1 & 0 & 0 & 0 & 0 & 0 \\
$H^{3}$ & 0 & 3 & 6 & 9 & 7 & 15 & 10 & 9 & 6 & 12 & 3 & 5 & 3 & 0 & 0 \\
$H^{4}$ & 3 & 9 & 21 & 19 & 20 & 47 & 27 & 25 & 29 & 42 & 20 & 17 & 13 & 6 & 1 \\
$H^{5}$ & 3 & 14 & 34 & 31 & 31 & 78 & 42 & 44 & 48 & 75 & 34 & 30 & 29 & 13 & 1 \\
$H^{6}$ & 2 & 9 & 18 & 25 & 23 & 50 & 31 & 34 & 28 & 52 & 19 & 23 & 22 & 9 & 4 \\
\hline
\end{tabular}

If we view $H_{\text {ét, }}^{k}\left(\mathcal{C}_{2,7}^{\text {glp }}, \mathbb{Q}_{\ell}\right)$ as a representation of $S_{7}$, then we may read off the values of the corresponding character $\chi_{k}$ from Table 1 as

$$
\chi_{k}(\sigma)=(-1)^{k}\left|\left(\mathcal{C}_{2,7}^{\mathrm{glp}}\right)^{F \sigma}\right|_{q^{6-k}}
$$

where $\left|\left(\mathcal{C}_{2,7}^{\text {glp }}\right)^{F \sigma}\right|_{q^{6-k}}$ denotes the coefficient of $q^{6-k}$ in the polynomial $\left|\left(\mathcal{C}_{2,7}^{\text {glp }}\right)^{F \sigma}\right|$.

It is a standard fact from representation theory of finite groups that the irreducible representations of $S_{m}$ are indexed by partitions of $m$. In Table 2 we decompose the cohomology groups $H_{\text {ét, } c}^{k}\left(\mathcal{C}_{2,7}^{\text {glp }}, \mathbb{Q}_{\ell}\right)$ into irreducible representations of $S_{7}$. A number $r$ in the column indexed by $7^{n_{7}} 6^{n_{6}} \cdots 2^{n_{2}} 1^{n_{1}}$ means that the irreducible representation corresponding to $7^{n_{7}} 6^{n_{6}} \cdots 2^{n_{2}} 1^{n_{1}}$ occurs with multiplicity $r$ in $H_{\text {ét,c }}^{k}\left(\mathcal{C}_{2,7}^{\text {glp }}, \mathbb{Q}_{\ell}\right)$.

\section{Eight points}

In this section, using techniques similar to those of the proof of Proposition 3.1, we show that the space of eight points in general position is minimally pure. We will also explain why similar techniques will not suffice to prove minimal purity for the space of eight points in general linear position (of course, this does not prove that the space of eight points in general linear position is not minimally pure).

Recall that eight points $P_{1}, \ldots, P_{8} \in \mathbb{P}^{2}$ are in general position if

(i) No three of the points lie on a line,

(ii) No six of the points lie on a conic, and

(iii) The eight points do not lie on a singular cubic with a singularity at one of the points $P_{1}, \ldots, P_{8}$.

We denote the space of octuples $\left(P_{1}, \ldots, P_{8}\right)$ of points in $\mathbb{P}^{2}$ in general position, up to projective equivalence, by $\mathcal{C}_{2,8}^{\mathrm{gp}}$.

Proposition 4.1 The space $\mathcal{C}_{2,8}^{\mathrm{gp}}$ of octuples of points in the plane in general position is minimally pure.

Proof The idea of the proof is the same as in the proof of Proposition 3.1: we construct a $\mathbb{F}_{q}$-rational and $S_{8}$-equivariant finite cover $\varphi: X \rightarrow \mathcal{C}_{2,8}^{\mathrm{gp}}$ such that $X$ is minimally pure, and the result will then follow (in fact, in this case, the cover will even be equivariant with respect to the much larger group $W\left(E_{8}\right)$, the Weyl group of the root system $\left.E_{8}\right)$.

To construct the space $X$ we observe that, if we blow up $\mathbb{P}^{2}$ in eight points $P_{1}, \ldots, P_{8}$ in general position, we obtain a geometrically marked Del Pezzo surface $S$ of degree 1 . Let 
$\pi: S \rightarrow \mathbb{P}^{2}$ denote the blow-down morphism. We rigidify the situation further by marking $S$ with an anticanonical curve $C$, i.e., a curve whose class is $3 L-E_{1}-\cdots-E_{8}$, where $L$ denotes the strict transform of a line and $E_{i}$ denotes the exceptional class corresponding to the point $P_{i}$. The curve $A=\pi(C)$ has degree 3 and passes through the points $P_{1}, \ldots, P_{8}$. Suppose that $A$ is reducible. Then $A$ contains a component $A_{1}$ of degree 1 . Since $P_{1}, \ldots, P_{8}$ are in general position, the component $A_{1}$ can contain at most two of the points. Thus, the residual curve $A_{2}$ must contain six of the points. But $A_{2}$ has degree 2 and is therefore allowed to contain at most five of the points. We conclude that $A$ must be irreducible.

Let $X$ be the space of triples $(S, B, C)$ such that $S$ is a Del Pezzo surface of degree $1, B$ is a geometric marking of $S$, and $C$ is an anticanonical curve on $S$ such that $A=\pi(C)$ is singular. Two triples $(S, B, C)$ and $\left(S^{\prime}, B^{\prime}, C^{\prime}\right)$ are equivalent if there is an isomorphism $S \rightarrow S^{\prime}$ taking the ordered basis $B$ to $B^{\prime}$ and the curve $C$ to $C^{\prime}$. The morphism $\varphi: X \rightarrow \mathcal{C}_{2,8}^{\mathrm{gp}}$ sending a triple $(S, B, C)$ to the octuple $\left(P_{1}, \ldots, P_{8}\right)$ defined by the blow-down of $B$ is clearly $\mathbb{F}_{q}$-rational, finite (of degree 12 ), and $S_{8}$-equivariant. It thus remains to show that $X$ is minimally pure.

The curve $A$ will be either a nodal or cuspidal cubic. Let $X^{\text {node }}$ denote the subspace of $X$ consisting of triples $(S, B, C)$ such that $A$ is nodal, and let $X^{\text {cusp }}$ be the subspace of $X$ consisting of triples such that $A$ is cuspidal. We then have that $X$ is the disjoint union

$$
X=X^{\text {node }} \sqcup X^{\text {cusp }} \text {. }
$$

The next step is to show that $X^{\text {node }}$ is isomorphic to a complement of a toric arrangement in an algebraic torus $T$ and that $X^{\text {cusp }}$ is isomorphic to a projectivization of a complement of a hyperplane arrangement in a vector space $V$ in such a way that $V$ can be naturally be identified with the tangent space of $T$ at the identity. This follows exactly as in Propositions $1.8,1.11$, and 1.17 of [19]. It now directly follows from Lemma 3.6 of [19] that there is a short exact sequence of mixed Hodge structures

$$
0 \rightarrow H_{\text {ét }, c}^{k}\left(X, \mathbb{Q}_{\ell}\right) \rightarrow H_{\text {ét, }}^{k}\left(X^{\text {node }}, \mathbb{Q}_{\ell}\right) \rightarrow H_{\text {ét, } c}^{k-1}\left(X^{\text {cusp }}, \mathbb{Q}_{\ell}\right)(-1) \rightarrow 0 .
$$

In particular, there is an inclusion

$$
H_{\text {ét }, c}^{k}\left(X, \mathbb{Q}_{\ell}\right) \hookrightarrow H_{\text {ét }, c}^{k}\left(X^{\text {node }}, \mathbb{Q}_{\ell}\right) .
$$

Since complements of toric arrangements are minimally pure, it follows that also $X$ is minimally pure. This completes the proof.

Remark 4.2 The cohomology groups of the space $X^{\text {cusp }}$ (at least before the projectivization) were computed as representations of the Weyl group $W\left(E_{8}\right)$ by Fleischmann and Janiszczak in [10]. The cohomology groups of $X^{\text {node }}$ were computed as representations of $W\left(E_{8}\right)$ by the author in [2]. Combining these two results using Lemma 3.6 of [19] gives the cohomology groups of $X$ as representations of $W\left(E_{8}\right)$.

Since the space of eight points in general position is isomorphic to the moduli space of geometrically marked Del Pezzo surfaces of degree 1, we can reformulate the above result as follows:

Corollary 4.3 The moduli space of geometrically marked Del Pezzo surfaces of degree 1 is minimally pure.

Thus, it is possible to determine the cohomology groups of $\mathcal{C}_{2,8}^{\mathrm{gp}}$ by counting points over finite fields. This is, however, rather nontrivial and the topic of ongoing research. 
One central aspect of the proofs of Propositions 3.1 and 4.1 has been the ability to find rational cubics through the points $P_{1}, \ldots, P_{m}$ in a consistent way. More precisely, we have in the general case been able to find rational cubics $A$ of fixed singularity type such that $\operatorname{Jac}(A) \cong \mathbb{G}_{m}$, and in the nongeneral case we have also had rational cubics $A$ of a single fixed singularity type but such that $\operatorname{Jac}(A) \cong \mathbb{G}_{a}$.

If we now consider eight points in general linear position, we can no longer achieve this type of consistency. For a general octuple $\left(P_{1}, \ldots, P_{8}\right)$, we will only have irreducible cubic curves passing through the points; i.e., there is no reducible cubic passing through them. However, as soon as seven of the points $\left(P_{1}, \ldots, P_{8}\right)$ lie on a conic, there will be no irreducible cubic passing through the octuple (otherwise the conic and the irreducible cubic would intersect at $\geq 7$ points, which contradicts Bézout's theorem). We can still cover $\mathcal{C}_{2,8}^{\text {glp }}$ with a space $X$ which decomposes as $X=\cup_{s \in S} X^{s}$ with each $X^{s} s$ isomorphic to a complement of an arrangement of tori or hyperplanes, but not in such a way that we can show that the covering map is proper and $X$ is minimally pure. More precisely, we either get a situation where $X$ clearly is minimally pure but the map is not proper, or a situation where the covering map is proper but the cohomological properties of the space $X$ are not so easily deduced. In the latter case, it is possible to compute the cohomology groups of the spaces $X^{s}$, so one possible way to go further in the pursuit of the cohomology of $\mathcal{C}_{2,8}^{\text {glp }}$ could be to try to compute the cohomology of the union using Gysin maps. This is, however, likely to be rather complicated, and it seems unlikely that the space $X$ will end up minimally pure. We remark in passing that Glynn's non-equivariant point count of $\mathcal{C}_{2,8}^{\text {glp }}$ is compatible with $\mathcal{C}_{2,8}^{\text {glp }}$ being minimally pure, but that it is still possible that an equivariant point count could rule out minimal purity.

The above being said, we can relax the conditions in the definition of octuples in general position a bit and still achieve minimal purity. More precisely, we can allow up to six of the points to lie on a conic and we can also allow one of the points to lie on a singularity of a cubic passing through the eight points (but we cannot allow both to happen for the same octuple). We can also use the techniques of the present paper to prove minimal purity for the space of eight points in "almost general position" in the sense of Dolgachev and Ortland; see [9], p. 67. This suggests that the answer to Question 1.3 is likely to be rather subtle, even in the simplest cases.

Authors' contributions

I thank Jonas Bergström, Frank Gounelas, Nathan Kaplan, Oliver Leigh, and Dan Petersen for interesting discussions and helpful comments, and two anonymous referees for many helpful comments and remarks.

Funding Open access funding provided by University of Gävle.

Received: 5 August 2020 Accepted: 21 October 2020 Published online: 11 November 2020

References

1. Bergström, J., Bergvall, O.: The equivariant Euler characteristic of $\mathcal{A}_{3}$ [2]. Annali della Scuola Normale Superiore di Pisa, Classe di Scienze, To appear, arXiv:1804.09628 (2018)

2. Bergvall, O.: Cohomology of complements of toric arrangements associated to root systems. arXiv:1601.01857 (2016)

3. Bergvall, O.: Equivariant cohomology of moduli spaces of genus three curves with level two structure. Geom. Dedic. 202, 165-191 (2019)

4. Bergvall, O.: Equivariant cohomology of the moduli space of genus three curves with symplectic level two structure via point counts. Eur. J. Math. 6, 262-320 (2020)

5. Bergvall, O., Gounelas, F.: Cohomology of moduli spaces of Del Pezzo surfaces. arXiv:1904.10249 (2019)

6. Das, R.: Arithmetic statistics on cubic surfaces. arXiv:2002.11183 (2020)

7. Das, R., O'Connor, B.: Configurations of noncollinear points in the projective plane. arXiv:1904.11409 (2019) 
8. Dimca, A., Lehrer, G.: Purity and equivariant weight polynomials. In: Lehrer, G.I. (ed.) Algebraic Groups and Lie Groups. Australian Mathematical Society Lecture Series, pp. 161-182. Cambridge University Press, Cambridge (1997)

9. Dolgachev, I., Ortland, D.: Point sets in projective spaces. Astérisque 165, 1-210 (1988)

10. Fleischmann, P., Janiszczak, I.: Combinatorics and Poincaré polynomials of hyperplane complements for exceptional Weyl groups. J. Combin. Theory Ser. A 63(2), 257-274 (1993)

11. Glynn, D.: Rings of geometries. II. J. Combin. Theory Ser. A 49(1), 26-66 (1988)

12. Gross, M., Hacking, P., Keel, S.: Moduli of surfaces with an anti-canonical cycle. Compos. Math. 151(2), 265-291 (2015)

13. lampolskaia, A., Skorobogatov, A., Sorokin, E.: Formula for the number of [9, 3] MDS codes. volume 41, pp. 1667-1671. Special issue on algebraic geometry codes (1995)

14. Kaplan, N., Kimport, S., Lawrence, R., Peilen, L., Weinreich, M.: Counting arcs in projective planes via Glynn's algorithm. J. Geom. 108(3), 1013-1029 (2017)

15. Kaplan, N., Matei, V.: Counting plane cubic curves over finite fields with a prescribed number of rational intersection points. arXiv:2003.13944 (2020)

16. Kisin, M., Lehrer, G.: Equivariant Poincaré polynomials and counting points over finite fields. J. Algebra 247(2), 435-451 (2002)

17. Kisin, M., Lehrer, G.: Eigenvalues of Frobenius and Hodge numbers. Pure Appl. Math. Q. 2(2), 497-518 (2016)

18. Looijenga, E.: Rational surfaces with an anticanonical cycle. Ann. Math. 114(2), 267-322 (1981)

19. Looijenga, E.: Cohomology of $\mathcal{M}_{3}$ and $\mathcal{M}_{3}^{1}$. In: Bödigheimer, C.-F. and Hain, R.M., (eds), Mapping Class Groups and Moduli Spaces of Riemann Surfaces, volume 150 of Contemporary Mathematics, pp. 205-228 (1993)

20. Ritzenthaler, C.: Problèmes arithmétiques relatifs à certaines familles de courbes sur les corps finis. PhD thesis, Université Paris Diderot-Paris 7 (2003)

\section{Publisher's Note}

Springer Nature remains neutral with regard to jurisdictional claims in published maps and institutional affiliations. 